\title{
Article \\ An Accurate Bandgap Voltage Reference Ready-Indicator
}

\author{
Rafailia Malatesta $^{1}$, George Souliotis ${ }^{1, *(1)}$, Fotis Plessas ${ }^{2}$ and Spyridon Vlassis ${ }^{3}$ \\ 1 Department of Electrical and Computer Engineering, University of the Peloponnese, 26334 Patras, Greece; \\ r.malatesta@go.uop.gr \\ 2 Department of Electrical and Computer Engineering, University of Thessaly, 38334 Volos, Greece; \\ fplessas@e-ce.uth.gr \\ 3 Electronics Laboratory, Department of Physics, University of Patras, 26504 Patras, Greece; \\ svlassis@physics.upatras.gr \\ * Correspondence: gsoul@uop.gr; Tel.: +30-2610369257
}

Citation: Malatesta, R.; Souliotis, G.; Plessas, F.; Vlassis, S. An Accurate Bandgap Voltage Reference

Ready-Indicator. Electronics 2022, 11, 723. https://doi.org/10.3390/ electronics 11050723

Academic Editor: Sai-Weng Sin

Received: 27 January 2022

Accepted: 24 February 2022

Published: 26 February 2022

Publisher's Note: MDPI stays neutral with regard to jurisdictional claims in published maps and institutional affiliations.

Copyright: () 2022 by the authors Licensee MDPI, Basel, Switzerland. This article is an open access article distributed under the terms and conditions of the Creative Commons Attribution (CC BY) license (https:// creativecommons.org/licenses/by/ $4.0 /)$.

\begin{abstract}
An accurate indication that the bandgap voltage reference (BGR) circuit is settled on its nominal value is essential in analog or mixed signal systems. In this paper, a generic method for accurate bandgap voltage reference (BGR) Ready-Indication (RI) is proposed. A RI signal shows that the BGR operates correctly but also is used to enable PoR circuits when those employ the bandgap voltage to generate the required thresholds. In many low power applications, several systems are periodically switched on and off to save power and to increase battery life. In such systems, most circuits must remain off, while BGR is not in ready mode, saving battery energy. In other critical applications, the Ready-Indicator can ensure that critical systems can be set on and the reference voltage is within the operating range. In this paper, the introduced methodology is applied to different reference voltage generators. Initially, a BGR with Ready-Indication is presented extensively, including post-layout simulations results in $22 \mathrm{~nm}$, while thereafter, the study of this method is extended in other BGR topologies. The universality of the proposed method is proved by verifying the operation in all the BGR topologies under study covering designs in $22 \mathrm{~nm}$ and $65 \mathrm{~nm}$ and with supply voltages $1.8 \mathrm{~V}, 1 \mathrm{~V}, 0.55 \mathrm{~V}$.
\end{abstract}

Keywords: bandgap voltage reference; bandgap voltage reference ready indicator; power-on-reset

\section{Introduction}

The Bandgap Voltage Reference (BGR) is a circuit widely used in most systems on chips producing a reference voltage, theoretically independent of the PVT variations. Older BGR topologies generate a constant voltage reference Vref equal to $1.25 \mathrm{~V}$, by the sum of the diode bandgap voltage and the thermal voltage multiplied by a factor. In more recent topologies, there has been an increasing interest in BiCMOS or CMOS circuits where the supply voltage tends to be under $1 \mathrm{~V}$ and the reference voltage must be below $1 \mathrm{~V}$. So far, very little attention has been given to the generation of an accurate Ready-Indication (RI) signal, indicating that Vref has reached and is locked in the nominal value. The RI signal is important in a system because it can be used to set the corresponding analog circuits in operating mode. In many recent low power applications, several systems are periodically switched on and off to save power and to increase battery life. In such systems, most circuits must remain off, while BGR is not in ready mode. If the circuits are enabled earlier or later than required, the overall system dissipates power, without being operatable. Currently, more often, a required RI signal or a reset (RST) signal can be generated by a Power-on-Reset (PoR) circuit, which, however, is not accurate and does not indicate whether a reference voltage is also settled and ready for use. Power-on-Reset is a circuit that provides a reset signal when the power supply is initiated and remains in a required voltage range [1,2]. The reset signal of a PoR circuit indicates whether the supply voltage is at a normal level so that the analog or mixed circuits are allowed to be enabled but not 
ensure that a reference voltage can be used. Most PoR systems are simple RC circuits [1], which, however, are not reliable due to large variations under several processes, voltage, and temperature conditions (PVT).

The PoR circuit and BGR ready indication are critical in analog and mixed signal circuits, only a few reliable solutions are found in the literature. In [1] a PoR circuit is presented, based on the supply voltage $V D D$ detection. The supply voltage used in that circuit is greater than $1.8 \mathrm{~V}$, but the threshold trigger voltage is highly sensitive to PVT variations. In [2] a more accurate, PoR system is proposed which is based on a bipolar transistor pair, similar to that of a BGR. Due to the bipolar transistors, the supply voltage that is used is relatively high greater than $3 \mathrm{~V}$. In [3] a hybrid bandgap reference is introduced which provides a voltage reference together with a PoR signal. The circuit has the advantage of high simplicity, but due to this, the reference voltage is sensitive over process and voltage variations and the PoR signal is not accurate. In [4] a BGR ready circuit is proposed for RFID application. The topology is based on two comparators to provide two logic signals about the status of the internal voltages of the specific BGR. However, details about the performance of this circuit under PVT variations are not given. A very simple BGR ready signal is produced in [5], but this is not accurate as it is based on the startup circuit of the BGR. In [6] a dedicated BGR for PoR circuit is introduced from 1.3 to $3 \mathrm{~V}$ supply voltage.

The drawback of the simple PoR circuits [1,2] is that they provide a reset signal only when VDD crosses the predefined thresholds, though the hybrid BGR-PoR circuits [3-6] provide a ready signal with a reference voltage. However, the suggested methods show a high sensitivity over PVT corners and usually dedicated solutions for specific applications are developed in each case. The major problem with producing an accurate RI is that other reliable voltage references that could be used for comparison are not available in the system.

In this paper, a generic method for producing accurate RI signals of the BGRs is proposed. The RI signal indicates that the BGR is settled and produces an accurate Vref which can be used by other circuits. The introduced method is based on the comparison of the voltage reference Vref with some other internal voltages produced by the proposed circuitry. Therefore, only a few additional comparators need to be employed with the BGR. The comparators required in this application can be implemented with simple operational amplifiers and the additional occupation in the layout area is small compared with the BGR core. Additionally, the circuitry generating RI signal does not affect the Vref itself and no noise or other interference is charging it. All comparisons with Vref are isolated through simple operational amplifiers without any feedback from RI to Vref.

The advantages of the introduced method are, first its high accuracy of RI signal, second, it can be employed with already known BGRs from the literature, and third, it can be used to improve even more the accuracy in other already proposed PoR circuits [3]. Another benefit is that with its flexibility it can be combined with already published in the literature BGR topologies and constitute a substantial solution to provide an accurate RI signal without decreasing the performance of an already efficient BGR. Additionally, using the RI signal for setting in off or in standby mode for other circuits, saves power consumption from the overall system. The cost paid for the extra circuit is the additional area and current consumption, but as already explained both are compensated by the corresponding advantages, the fact that the area is too small, and the overall current consumption of the system can be reduced.

The rest of the paper is organized as follows: In Section 2, the BGR operation during powerup or down and design approach of BGR RI is discussed. In Section 3, circuit designs and simulation results are presented for several BGR topologies. The conclusions are given in Section 4. 


\section{The Design Approach of BGR RI}

During the power-up procedure, the BGR is one of the first circuits starting to operate and must be ready in a short time to provide the reference voltage in the rest circuits of a system, among them in the PoR circuit. During power-up or down the supply voltage $V D D$ takes a ramp-up or down form, approximately, as shown in Figure 1. During these time slots, BGR provides an uncertain reference voltage Vref, especially when the supply voltage $V D D$ is not yet in the nominal range. In that case, Vref should be ignored from the rest of the system and a RI signal should decline the start-up of the rest circuits. Usually, a start-up circuit is used in the BGR circuit to ensure that it will start operating normally and will not be latched in off state. In some cases, the start-up circuit could be used as a BGR ready indicator, but this is not enough for an accurate RI signal. The reason is that, for a considerable timeframe, the start-up circuit remains active, while the BGR core internal nodes have already been self-biased, resulting in an uncertain value of Vref, as shown in Figure 1. Based on this procedure, a high risk of detecting a possible fault BGR operation mode exists. Under these conditions, Vref is insufficient to be used as a standalone signal to certify that BGR operates in a normal state and that $V D D$ has reached its nominal value. Then an appropriate comparison between $V r e f, V D D$, and some internal voltages must be performed in order to produce an accurate RI signal.

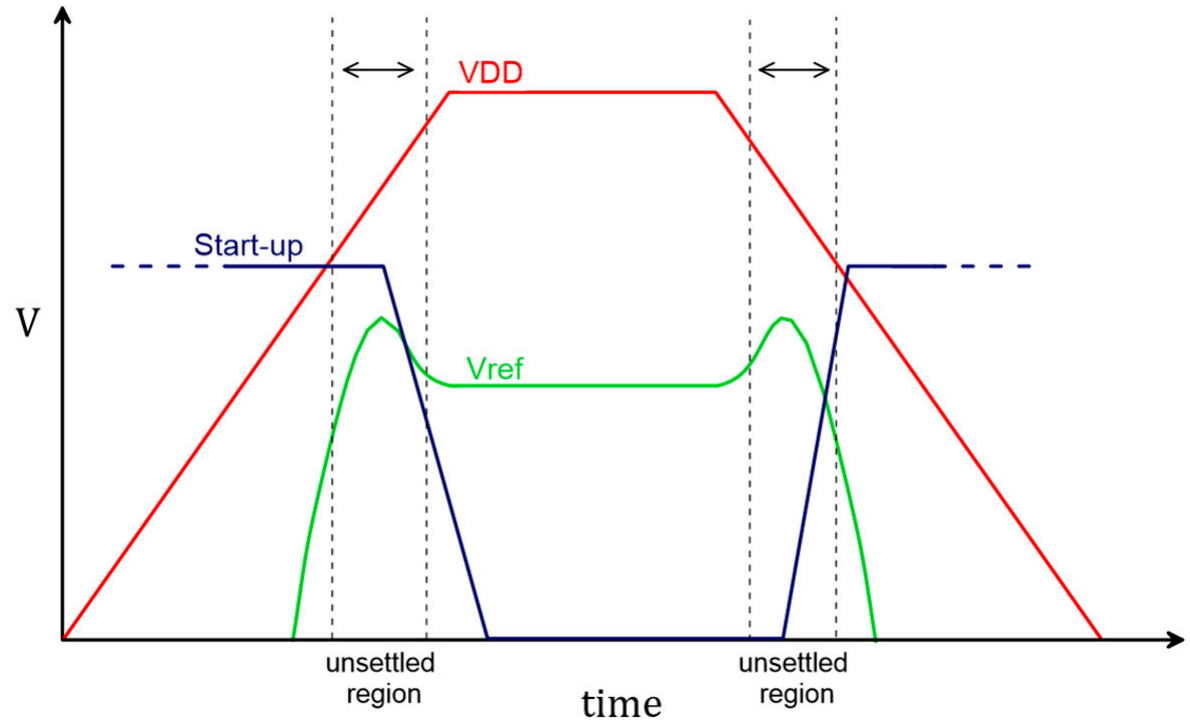

Figure 1. Vref and start-up during power-up and power-down.

The concept of the required comparisons is depicted in the simplified block diagram of Figure 2. The internal voltages must be selected carefully, and they must be related to Vref and $V D D$ through a known correlation. In Figure 2, Vref is the generated reference voltage, $V d$ is a proportional voltage to $V D D$, and stup is a voltage generated by the startup circuit. For most cases, two to three voltage comparisons, between these voltages, are required. The number of comparisons depends on the supply voltage, while the lower voltage headroom makes the procedure harder. It is important that neither voltage nor current can be considered as known or constant during the power-up or power-down procedure, and the comparator's bias depends on the specific state.

Towards these conditions, the following comparisons must be performed,

- Comparison between Vref and $V d$ (proportional to $V D D$ )

- Comparison between Vref and stup (start-up status)

- Comparison between stup and $V d$ (this step is usually needed in low voltage circuits)

However, it must be noted that depending on the particular BGR topology, a similar signal could be utilized instead of one of these described in the steps above. In the following presented topologies, these steps are applied to produce an RI signal. 


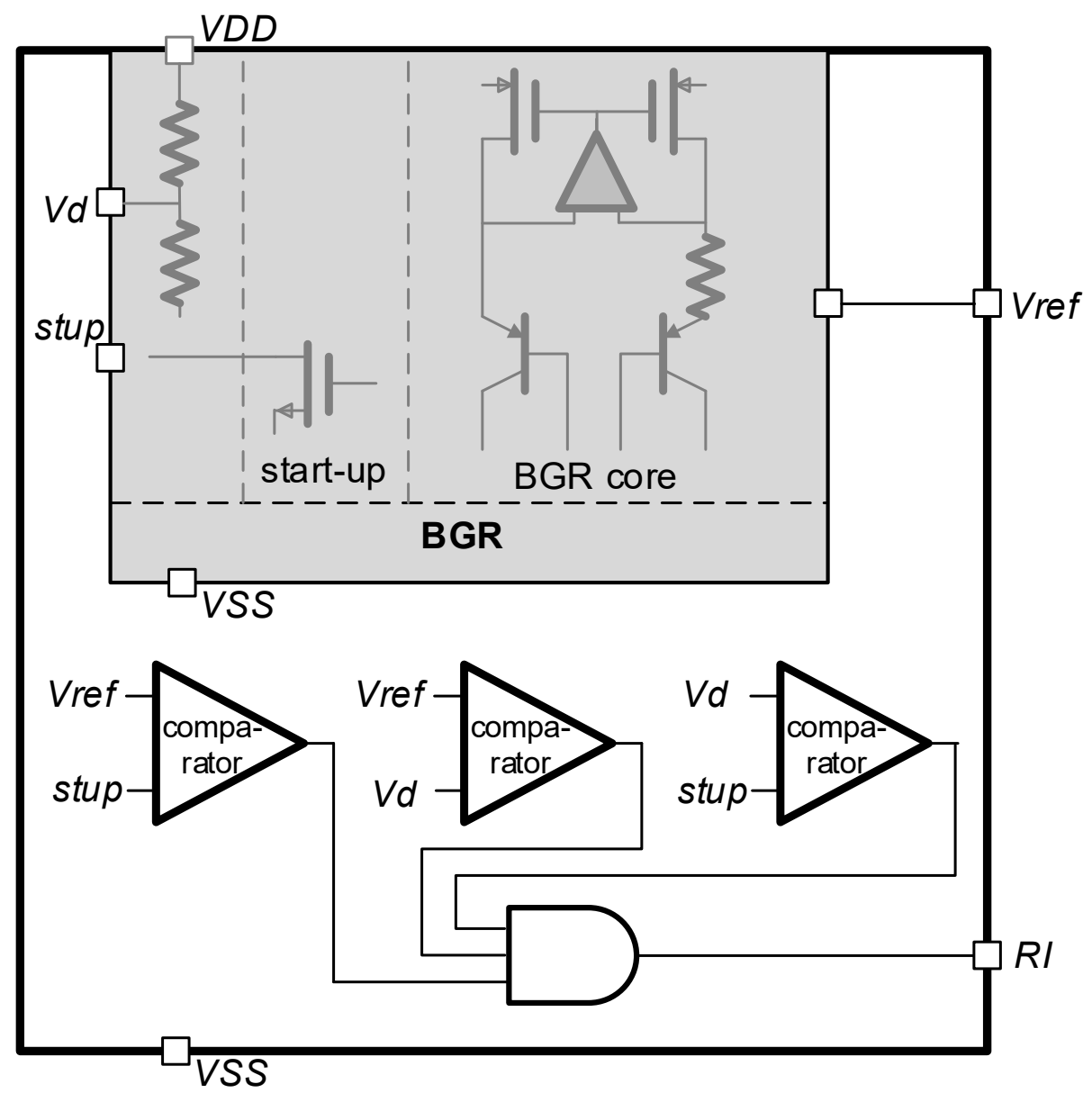

Figure 2. Simplified block diagram of the proposed method.

\section{Circuit Design and Simulation Results}

The abovementioned observations and obstacles are taken under consideration and applied in three separate topologies, so as the general proposed method to be proved. The first RI circuit has been employed in a BGR topology [7] shown in Figure 3. This circuit has been designed and simulated with a $22 \mathrm{~nm}$ Fully Depleted Silicon On Insulator (FDSOI) technology node and $1.8 \mathrm{~V}$ supply voltage and is presented in Section 3.1, as BGR\#1 (22 nm, $1.8 \mathrm{~V})$. The same topology of [7] has been also designed and tested in a $65 \mathrm{~nm}$ CMOS technology node with $1 \mathrm{~V}$ supply voltage and is presented in Section 3.2, as BGR\#1 (65 nm, $1 \mathrm{~V})$. Moreover, other BGR topologies [3,8], with different operating conditions have been investigated in order to verify the proposed methodology for the RI. A two operational amplifier based BGR [8] has been designed in CMOS $65 \mathrm{~nm}$ technology node and $1 \mathrm{~V}$ supply voltage and is presented in Section 3.3, as BGR\#2 (65 nm, $1 \mathrm{~V})$. Finally, a hybrid PoR-BGR circuit [3] has been designed in CMOS $65 \mathrm{~nm}$ and $0.55 \mathrm{~V}$ supply voltage and is presented in Section 3.4, as BGR\#3 (65 nm, $0.55 \mathrm{~V})$.

The proposed BGR circuits have been tested under several power-on and power-off conditions, where the supply voltage $V D D$ is sequentially on and off, through a ramp function with different rise, fall, and settling times at different final voltage levels. The selected temperature ranges, used for the corner simulation results in each design, are extended close to the corners, offered by the corresponding technology for model operation and layout limitations. 


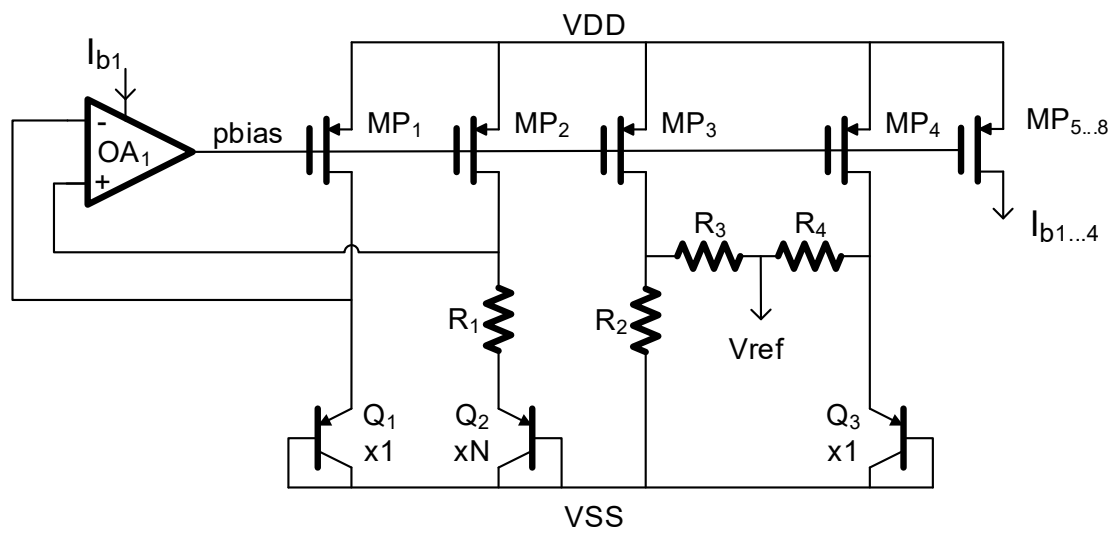

(a)

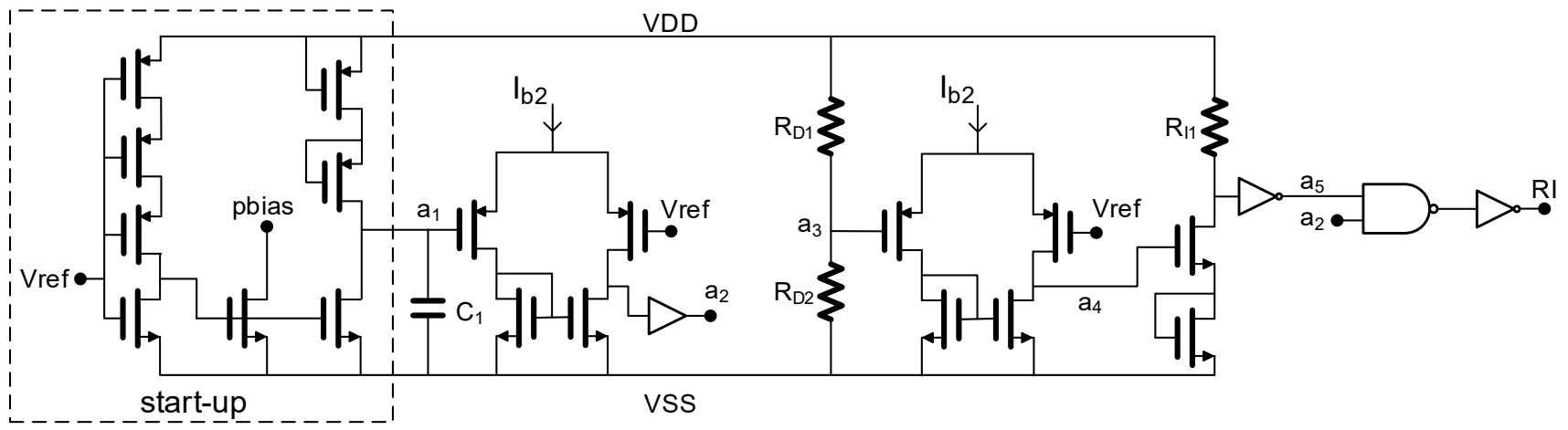

(b)

Figure 3. BGR\#1 in FDSOI $22 \mathrm{~nm}$ and $1.8 \mathrm{~V}$ supply voltage (a) core circuit and (b) RI circuit.

\subsection{RI Circuit for BGR\#1 in $22 \mathrm{~nm}, 1.8 \mathrm{~V}$ Supply Voltage}

For the first BGR [7] core with the proposed BGR RI circuit, a combination of internal signals is used, being compared with Vref and resulting in the RI signal, as shown in Figure 3. The main comparison is realized between Vref and the $a 1$ signal generated by the start-up circuit. This comparison ensures that the main bias of the BGR core has already started, and the state of the start-up circuit is off. This comparison, however, is not sufficient because, Vref could be in a lower level than $a 1$ but not yet in its nominal value, as the start-up is not in a clear on or off state. A possibly lower than the nominal $V D D$ could make the BGR start, the start-up circuit be in an off state, but Vref to be in an unsettled value. Therefore, an additional comparison ensuring that VDD is within the acceptable range must be performed. The indicative graph of Figure 1 depicts the unsettled regions where the RI must properly indicate that BGR does not operate in its normal state. A comparison is required between Vref and VDD, as well as between Vref and $a 1$ generated by the start-up circuit.

The BGR\#1 of Figure 3 has been designed, initially, in a $22 \mathrm{~nm}$ FDSOI technology node. Post-layout simulations have been performed to validate the BGR and RI operation. The BGR is tested under several power-up and power-down conditions, where the supply voltage $V D D$ is sequentially on and off, through a ramp function with different rise and fall times and settling at different final levels. The ramp function has been employed to simulate the way a supply voltage is applied on the circuits in a chip.

Post-layout simulation results depicted in Figure 4 show the RI, the internal net voltages required for the comparison, Vref voltage, and VDD. Vref takes unpredictable values before settling at the nominal value, as shown in Figure 4a. It is also showing that the rising part of the waveform of the internal net $a 1$ is different than the falling part. The asymmetry is due to the internal capacitances and different internal resistance 
and switching times during powering up or down. The waveforms could be even more different in PVT corners and the correct detection even harder. Additionally, from corner simulations, it can be shown that the critical voltages not only take different final values but also can be of highly different waveforms with different rise and fall times and different settling times. Therefore, the two comparisons, one of Vref with a3, proportional to VDD and the other of Vref with a1, guarantee the correct RI detection. The capacitor at node a1 prevents the corresponding voltage from glitches and provides the necessary delay, during power-up and power-down. It must be noted that the Vref also, is simulated with a capacitive load, about $8 \mathrm{pF}$.

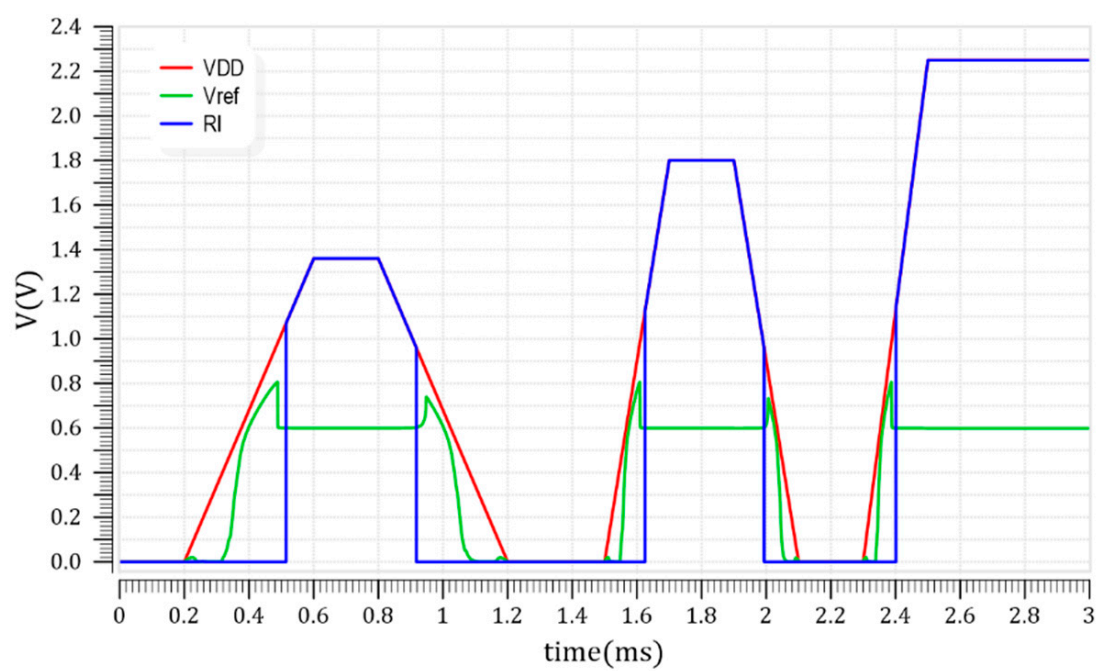

(a)

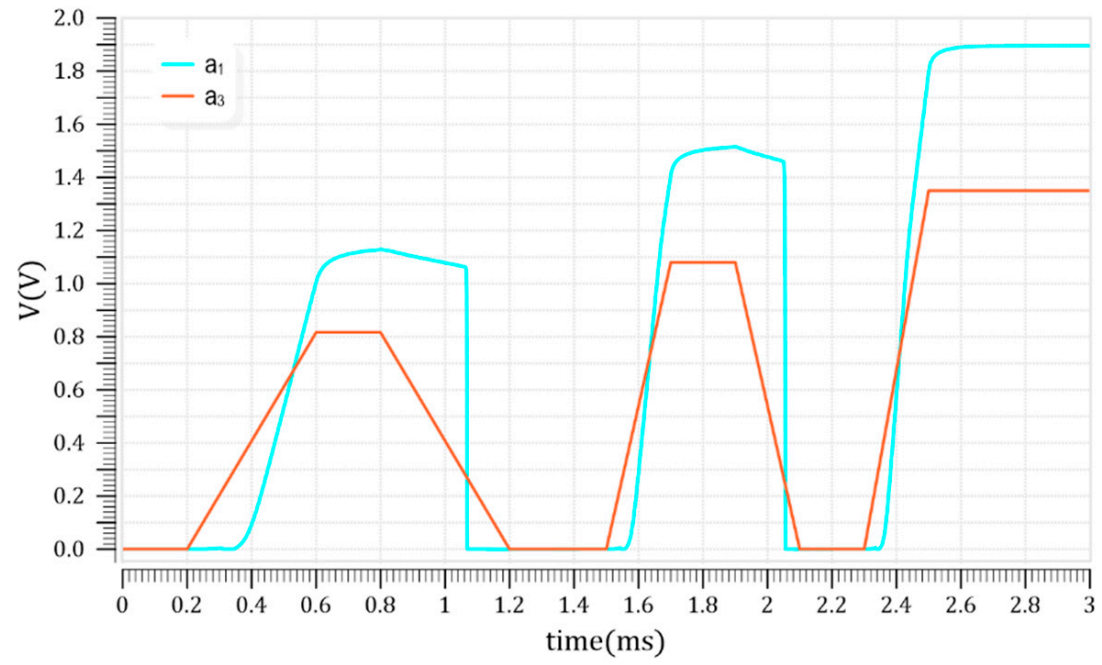

(b)

Figure 4. The BGR\#1 (22 nm, $1.8 \mathrm{~V})$ Ready-Indication for different power-up and power-down conditions at a typical temperature $\left(27^{\circ} \mathrm{C}\right)$. (a) Important input-output voltages and (b) internal critical voltages used for comparison.

Post-layout corner simulations results are shown in Figure 5. The RI detects clearly whether Vref is in the nominal value. In Figure 5b, the slopes of Ready-indication, close to the edges, are due to the $V D D$ value. In those cases, $V D D$ has not reached the final values and so, the Ready-indication has the same values, following VDD ramp-up or ramp-down. 


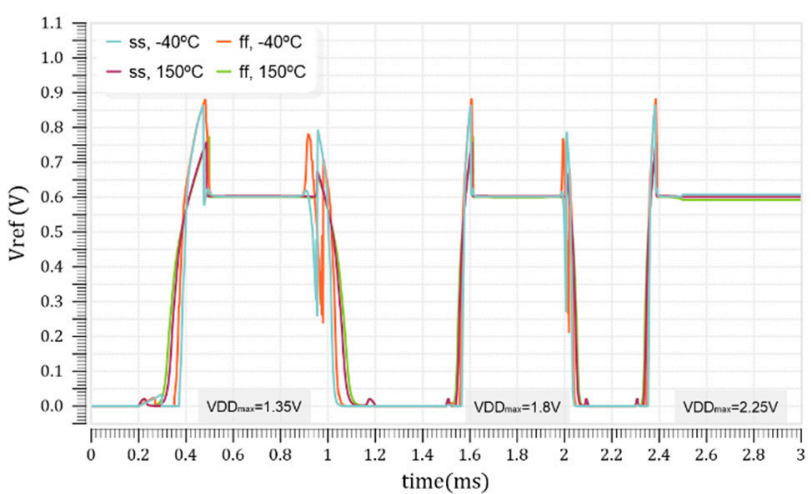

(a)

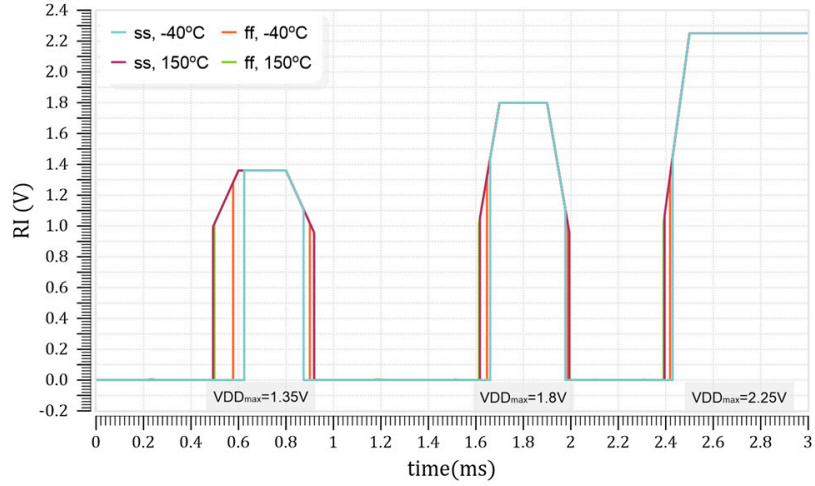

(b)

Figure 5. Simulation results for BGR\#1 (22 nm, $1.8 \mathrm{~V})$ in PVT corners. (a) Vref, (b) RI.

The corner analysis has been performed for the worst process corners, fast-fast (ff), and slow-slow (ss) and temperatures $-40^{\circ} \mathrm{C}$ and $150{ }^{\circ} \mathrm{C}$.

\subsection{RI Circuit for BGR\#1 in $65 \mathrm{~nm}, 1 \mathrm{~V}$ Supply Voltage}

The core of BGR presented in this subsection is of the same topology as that presented in Section 3.1 and Figure 3a. The difference now is that it has been designed and simulated in a $65 \mathrm{~nm}$ CMOS technology, with a $1 \mathrm{~V}$ supply voltage. Utilizing a $1 \mathrm{~V}$, instead of $1.8 \mathrm{~V}$ supply voltage, results in a more complicated procedure to achieve the correct Ready-Indication, due to the limited voltage headroom in the transistors. Following the implementation proposed in Section 3.1 with two comparators, the requested accuracy for RI is not achieved. Consequently, to avoid fault indication, one more comparison is required between $a 1$ and $a 3$, as shown in Figure 6 . The voltage $a 1$ is produced by the start-up circuit and $a 3$ is proportional to $V D D$.

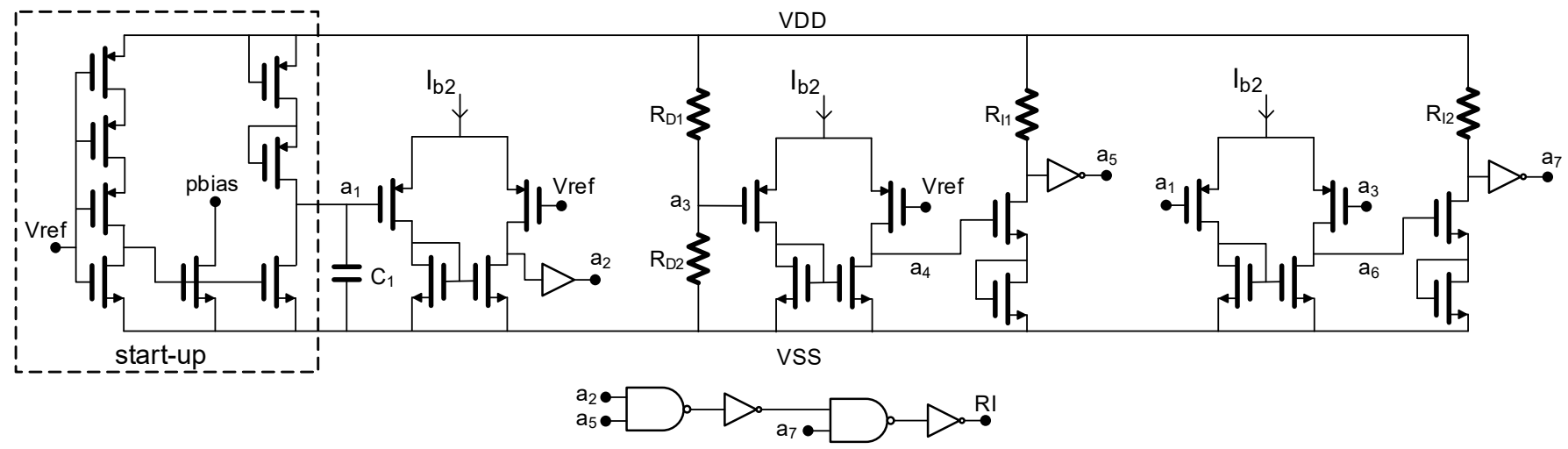

Figure 6. The RI circuit for BGR\#1 in CMOS $65 \mathrm{~nm}$ technology and $1 \mathrm{~V}$ supply voltage.

Simulation results presented in Figure 7, show the Vref voltage, as it is generated by the transitions of $V D D$ during power-up and power-down in different levels of $V D D$. Additionally, the RI signal is depicted in the same figure. Vref takes unsettled values before and after settling at the nominal value, alike to the results of the previous implementation. Therefore, the appropriate attention has been focused on these parts, considering that RI is detected clearly when Vref is within the nominal value. 


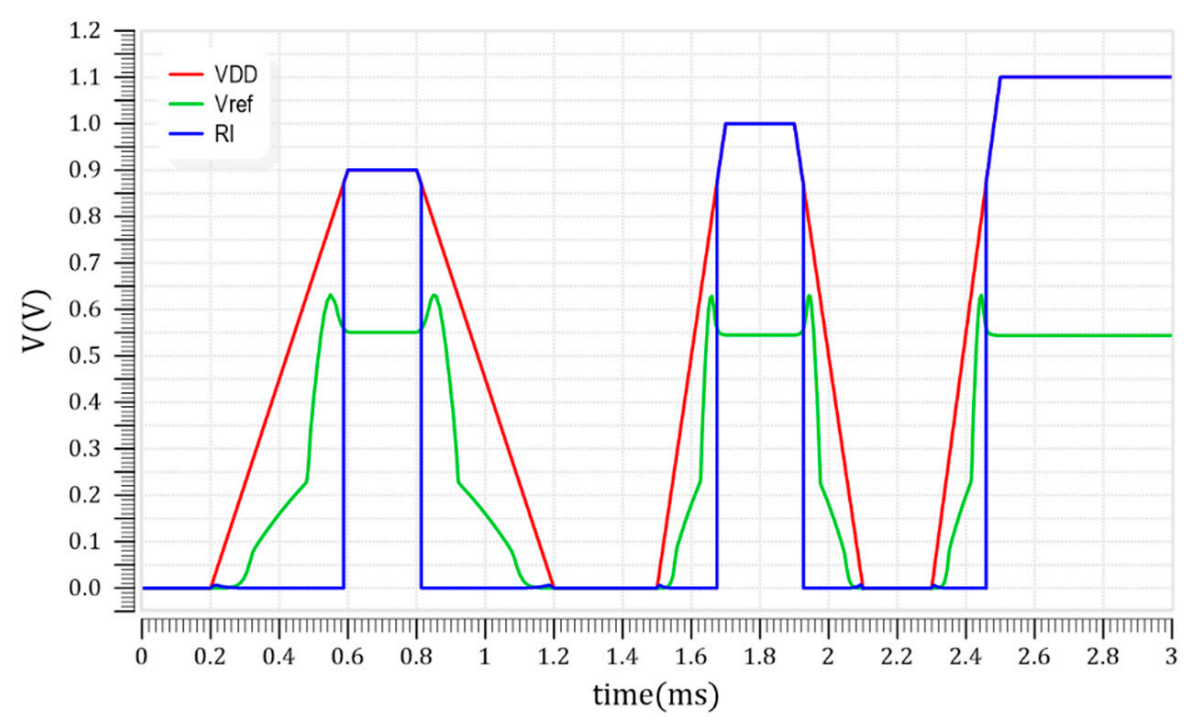

Figure 7. Simulation results for BGR\#1 $(65 \mathrm{~nm}, 1 \mathrm{~V})$ in typical temperature $\left(27^{\circ} \mathrm{C}\right), 1 \mathrm{~V}$ supply voltage, $\pm 10 \%$.

The precise detection of the RI becomes even harder in PVT corners, which constitute the final step of the procedure for the last settings to take place. Corner simulation results are shown in Figure 8, where corner analysis has been performed for the process corners ss and ff, with $V D D$ equal to $1 \mathrm{~V}$, supply voltage variation $\pm 5 \%$, and temperatures $-40{ }^{\circ} \mathrm{C}$ and $+120{ }^{\circ} \mathrm{C}$.

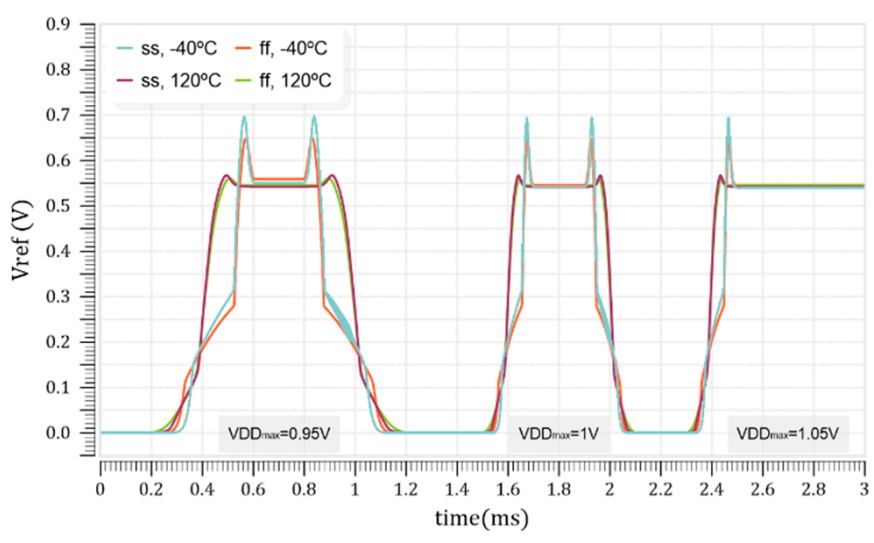

(a)

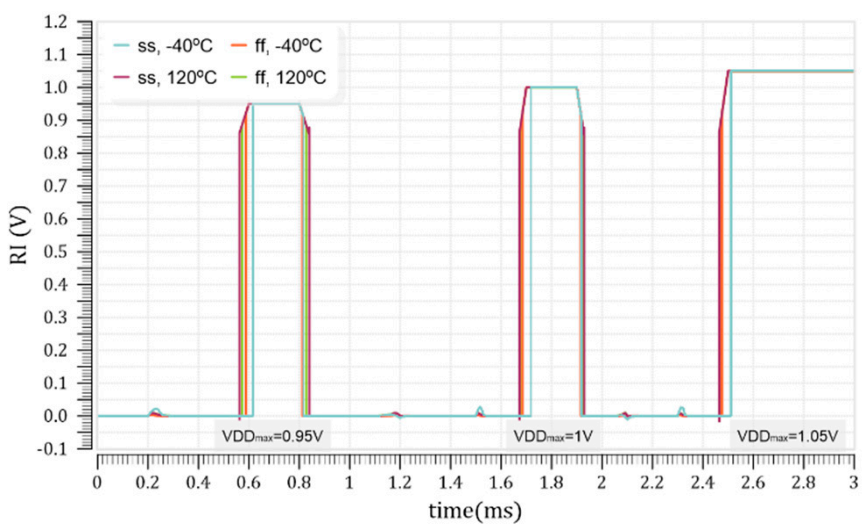

(b)

Figure 8. Simulation results for BGR\#1 (65 nm, 1 V) in PVT corners. (a) Vref, (b) RI.

\subsection{Ready Indicator for BGR\#2 in $65 \mathrm{~nm}, 1 \mathrm{~V}$ Supply Voltage}

As mentioned above, in an attempt of further investigation of the RI concept, more BGR circuits with various features have been tested. The BGR\#2 in this section is a two operational amplifiers topology, presented in [8] and has been modified as shown in Figure 9, to generate an RI signal. The BGR has been designed in a CMOS $65 \mathrm{~nm}$, operating with a $1 \mathrm{~V}$ supply voltage. 


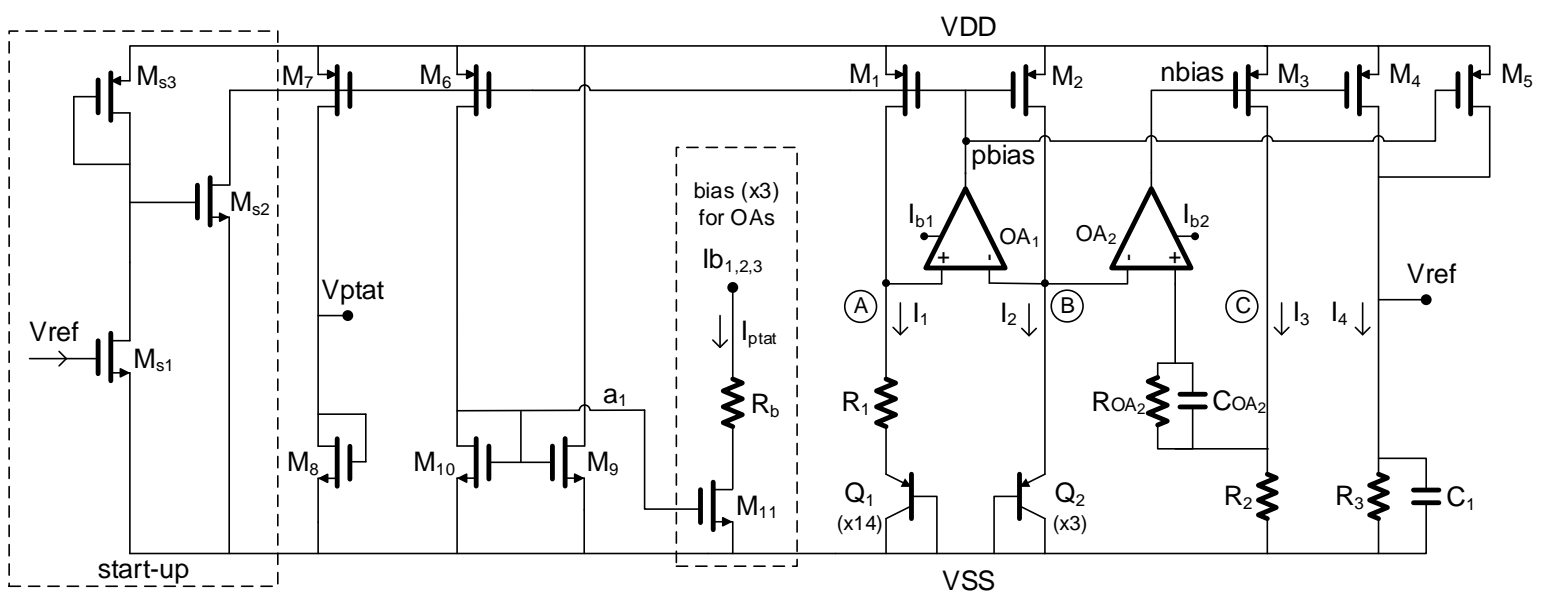

(a)

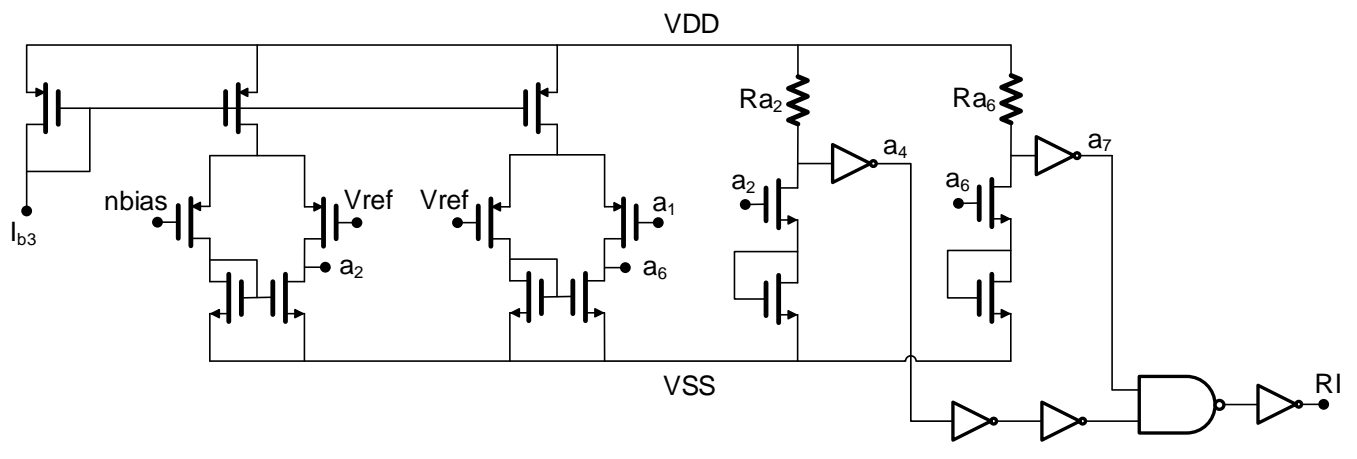

(b)

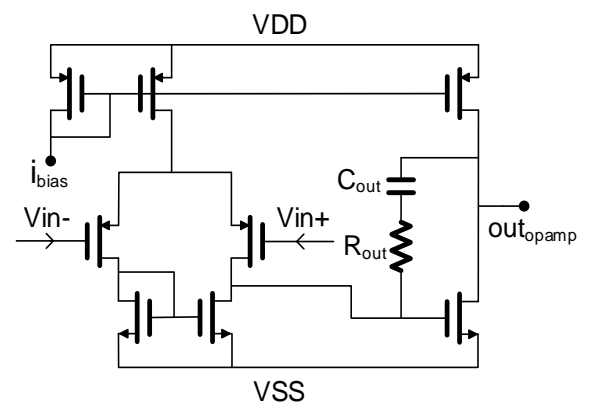

(c)

Figure 9. BGR\#2 in CMOS $65 \mathrm{~nm}$ technology, $1 \mathrm{~V}$ supply voltage (a) core circuit (b) RI circuit and (c) the operational amplifier circuit.

In the present case, the contribution of only two comparators for the RI generation is sufficient. As has been previously reported, the first comparison occurs between Vref and start-up (signal $a 1$ in Figure 9). The second comparison is taken between Vref and the output nbias of the second operational amplifier in Figure 9.

Simulation results that are presented in Figure 10, show Vref, as it is generated during the transitions of $\operatorname{VDD}(1 \mathrm{~V}, \pm 10 \%)$, in comparison with the RI signal. PVT corner simulations results are shown in Figure 11, where corner analysis has been performed for the process corners ss and ff, with $V D D$ equal to $1 \mathrm{~V}$, supply voltage variation $\pm 5 \%$, and temperature $-40^{\circ} \mathrm{C}$ and $+120^{\circ} \mathrm{C}$. 


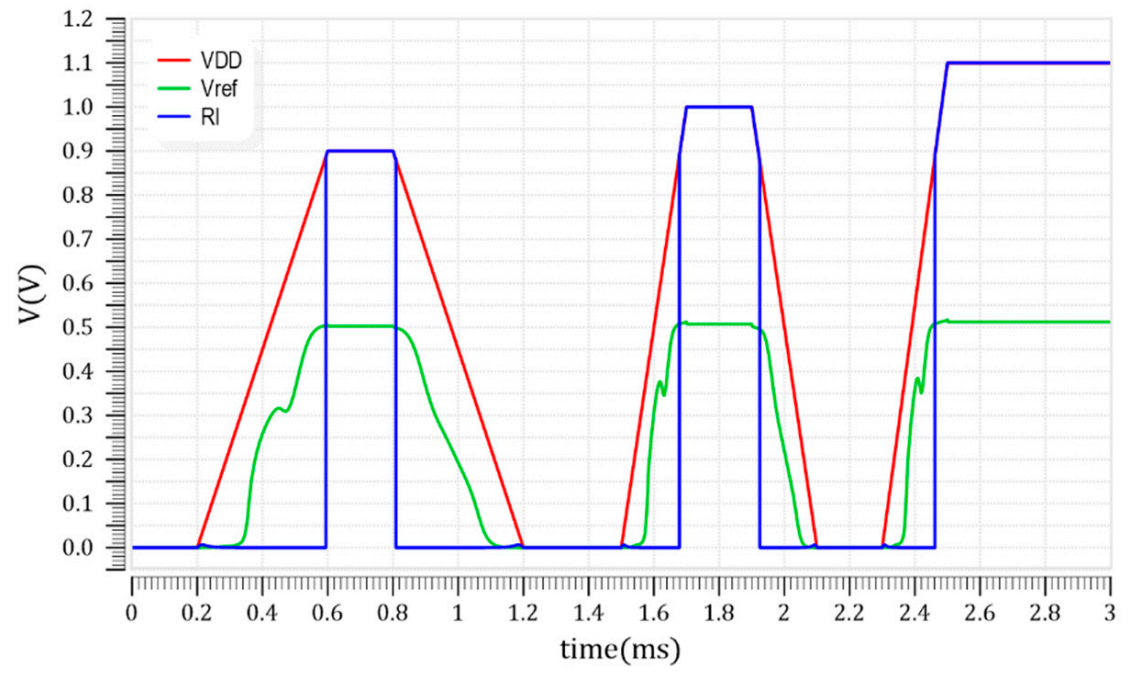

Figure 10. Simulation results for BGR\#2 $(65 \mathrm{~nm}, 1 \mathrm{~V})$ in typical temperature $\left(27^{\circ} \mathrm{C}\right)$ and $1 \mathrm{~V}$ supply voltage, $\pm 10 \%$.

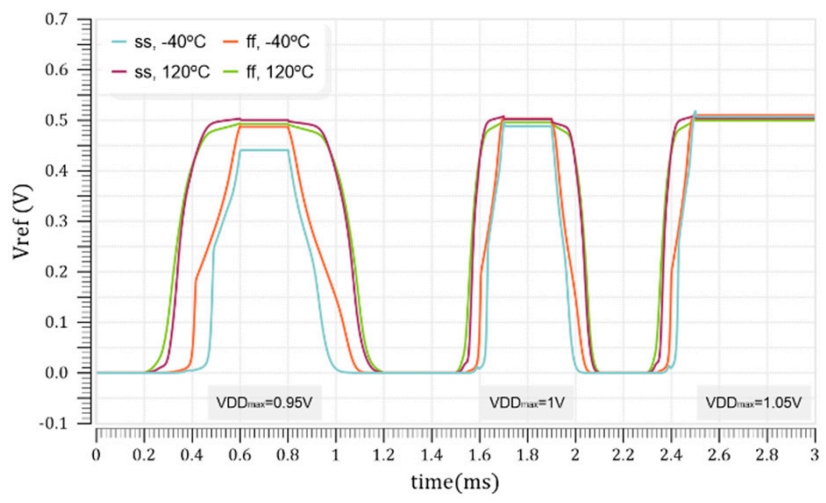

(a)

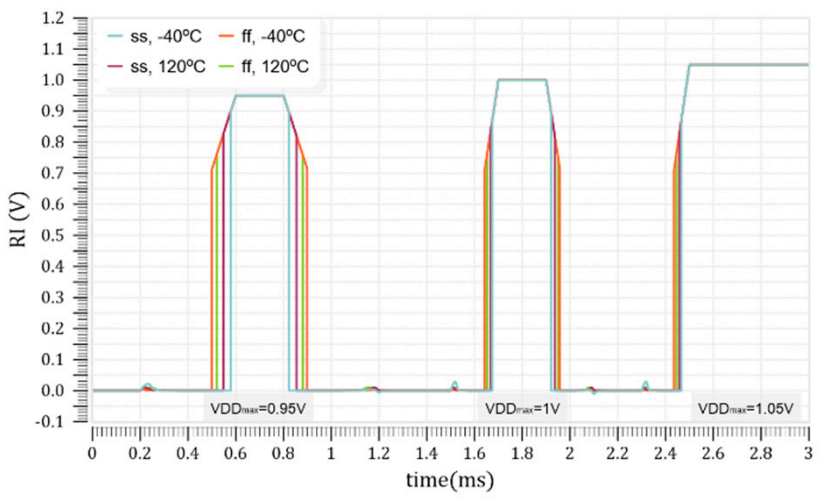

(b)

Figure 11. Simulation results for BGR\#2 (65 nm, 1 V) in PVT corners. (a) Vref, (b) RI.

\subsection{Ready Indicator for BGR\#3 in $65 \mathrm{~nm}, 0.55 \mathrm{~V}$ Supply Voltage}

A dedicated circuit for PoR with BGR is presented in [3]. In this paper, a modified topology for higher accuracy in RI is proposed. The BGR\#3 operation is based on MOS transistors only, without the contribution of bipolar transistors. It has been modified, as shown in Figure 12, and has been designed and simulated in a $65 \mathrm{~nm}$ CMOS technology with $550 \mathrm{mV}$ supply voltage. By reducing the value of the supply voltage to $550 \mathrm{mV}$, similarly to the previous circuits, the circumstances which should be taken under consideration for the achievement of precision become highly demanding. On the other hand, the most significant advantage of this circuit is the low power consumption, while the main disadvantage is the slow power-up and power-down time, which is expected in circuits with low current bias. Concerning the particular circuit, a relevant study for the suggested method has been conducted in [3], to address the issue of RI. The RST signal in that paper represents the expected RI signal. However, simulation results regarding the chosen technology depicted in Figure 13, show that the RST output works while the Vref is out of range. For more accurate operation a modified version of this circuit is proposed, by adding a comparison between $h_{\text {out }}$ and $g_{\text {in }}$ signals. 


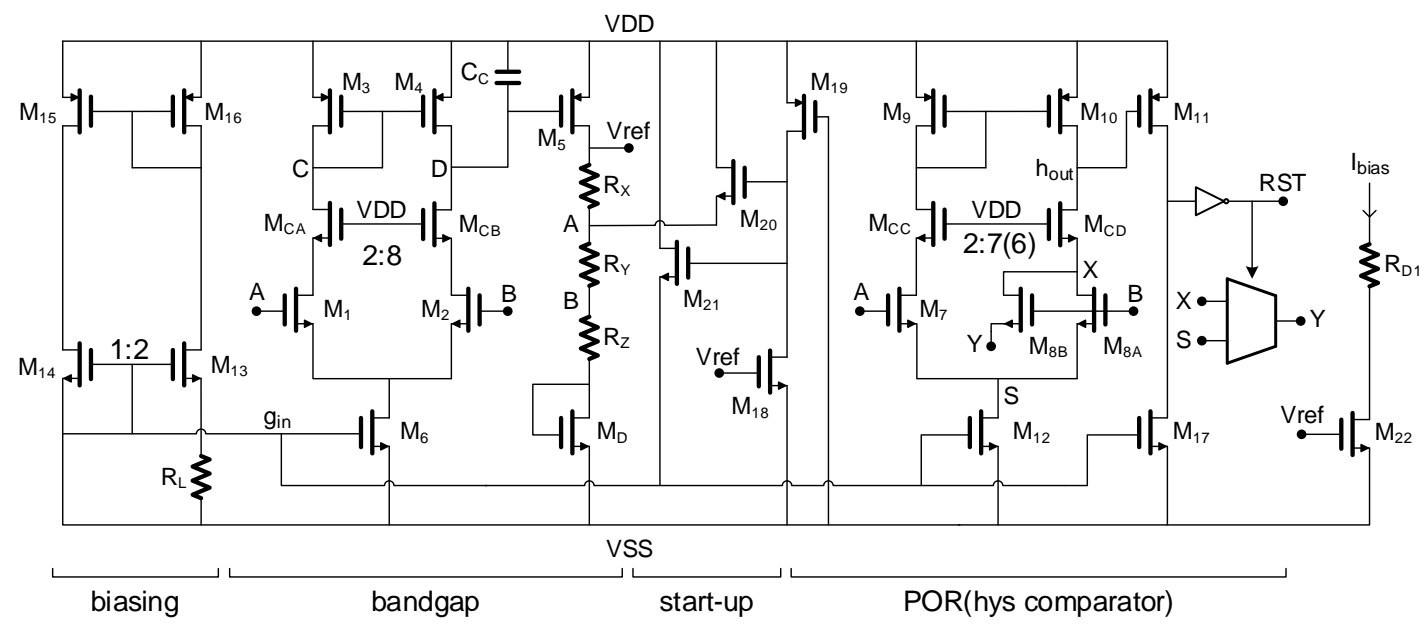

(a)

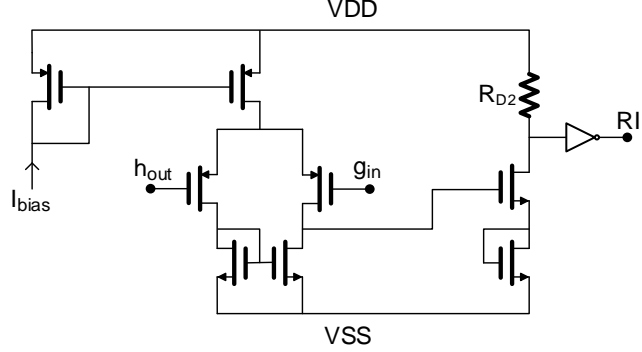

(b)

Figure 12. BGR\#3 in CMOS $65 \mathrm{~nm}$ technology, $0.55 \mathrm{~V}$ supply voltage (a) core circuit (b) RI circuit.

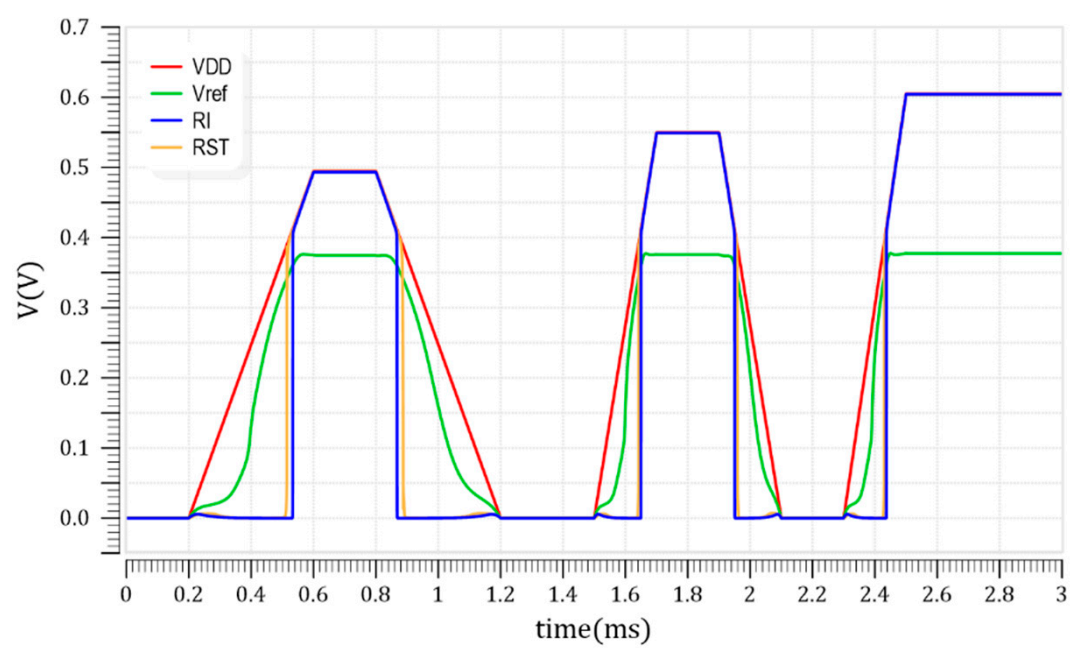

Figure 13. Simulation results for BGR\#3 $(65 \mathrm{~nm}, 550 \mathrm{mV})$ in typical temperature $\left(27^{\circ} \mathrm{C}\right), 550 \mathrm{mV}$ supply voltage, $\pm 10 \%$.

Simulation results of the proposed method on BGR\#3, which are presented in Figure 13, show improvement in RI compering with the already existing RST signal. The RI and Vref voltages are depicted and generated during the transitions of $V D D(550 \mathrm{mV}, \pm 10 \%)$. This BGR has been tested in corner analysis as well, showing correct operation and improved accuracy. PVT Corner simulations results are shown in Figure 14, where corner analysis has been performed for the process corners ss and ff, with $V D D$ equal to $550 \mathrm{mV}$, supply voltage variation $\pm 5 \%$, and temperature $-40{ }^{\circ} \mathrm{C}$ and $+120{ }^{\circ} \mathrm{C}$. From simulation results, it is shown that Vref is not constant over variations of process corners. This behavior is 
reported, also, in the original work in [3]. However, while Vref is considered to operate at acceptable levels, RI also gives a correct positive signal.

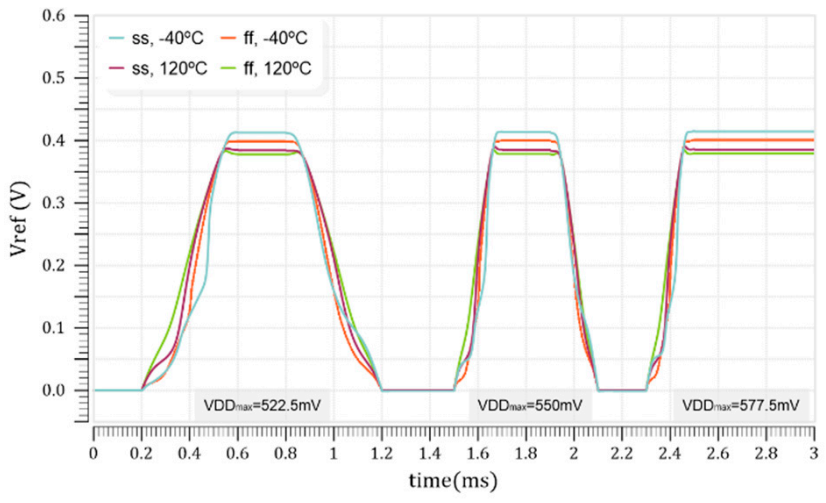

(a)

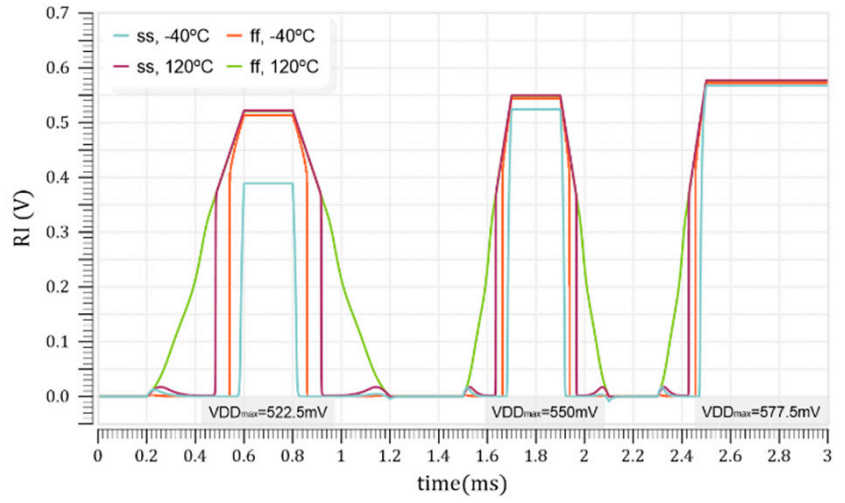

(b)

Figure 14. Simulation results for BGR\#3 $(65 \mathrm{~nm}, 550 \mathrm{mV})$ in PVT corners. (a) Vref, (b) RI

\section{Conclusions}

A step by step method for generating a BGR Ready-Indication (RI) has been presented in this paper. It offers an accurate ready indication of whether the reference voltage is within a short range of the nominal value; a significant advantage is that it can be applied in different BGR topologies, covering low or high voltage BGRs, low or high consumption BGRs, and topologies with or without bipolar transistors. The RI signal can replace the reset signal offered by the PoR circuits which does not ensure that the reference voltage is already in the nominal value. Therefore, the method is suitable for improved PoR circuits providing a more accurate reset signal. The presented method is based on the comparison of the BGR voltage reference with internal voltages without requiring other external voltage references. In this paper, the results of extended post-layout simulations of a BGR designed in a FDSOI $22 \mathrm{~nm}$ technology and other BGR topologies in a CMOS $65 \mathrm{~nm}$ technology validate that the proposed method is accurate and generic. It has been also applied to a hybrid BGR-PoR circuit reported in the literature, to verify that it can improve the accuracy of the corresponding reset signal of existing PoR circuits. The performance of the suggested circuit in each BGR of hybrid PoR-BGR topology is verified from simulation results obtained with extended temperature range and setting the supply voltage sequentially in different levels.

Author Contributions: Conceptualization, R.M., G.S. and F.P.; methodology, R.M., G.S.; software, R.M., G.S.; validation, R.M., G.S. and F.P.; formal analysis, R.M.; investigation, R.M., G.S. and F.P.; resources, R.M., G.S., F.P. and S.V.; data curation, R.M., G.S., F.P. and S.V.; writing-original draft preparation, R.M., G.S.; writing-review and editing, R.M., G.S., F.P. and S.V.; visualization, R.M.; supervision, G.S. and F.P.; project administration, F.P.; All authors have read and agreed to the published version of the manuscript.

Funding: This research received no external funding.

Data Availability Statement: All data are included within the manuscript.

Conflicts of Interest: The authors declare no conflict of interest.

\section{References}

1. Prakash, R. Zero quiescent current, delay adjustable, power-on-reset circuit. In Proceedings of the 2014 IEEE Dallas Circuits and Systems Conference (DCAS), Richardson, TX, USA, 12-13 October 2014; pp. 1-4.

2. Zhang, J.; Jiang, L.; Zeng, Z. Design of a Novel Power-on-Reset Circuit Based on Power Supply Detector. In Proceedings of the 2009 International Conference on Scalable Computing and Communications, Eighth International Conference on Embedded Computing, Dalian, China, 25-27 September 2009; pp. 355-359.

3. Zhou, B.; Liu, S.; Chiang, P. Low-complexity $0.55-\mathrm{V} 2.5-\mu \mathrm{W}$ bandgap reference and power-on reset hybrid circuit. Electron. Lett. 2016, 52, 346-348. [CrossRef] 
4. Ochoa, A. Bandgap Ready Circuit. Patent US20120313665A1, 13 December 2012.

5. Kamel, A.I.; Ahmed, S.; Lee, S.S. A high wide band PSRR and fast start-up current mode bandgap reference in $130 \mathrm{~nm}$ CMOS technology. In Proceedings of the 2016 IEEE International Symposium on Circuits and Systems (ISCAS), Montreal, QC, Canada, 22-25 May 2016.

6. Lazar, A.; Florea, M.; Burdia, D.; Lazar, L.; Lazar, G.; Butnicu, D. A bandgap reference circuit design for Power-on Reset related circuits. In Proceedings of the 2009 International Symposium on Signals, Circuits and Systems, Iasi, Romania, 9-10 July 2009; pp. 1-4.

7. Doyle, J.; Young, J.L.; Yong, B.K.; Wilsch, H.; Lombardi, F. A CMOS subbandgap reference circuit with 1-V power supply voltage. IEEE J. Solid-State Circuits 2004, 39, 252-255. [CrossRef]

8. Souliotis, G.; Plessas, F.; Vlassis, S. A high accuracy voltage reference generator. Microelectron. J. 2018, 75, 61-67. [CrossRef] 